\title{
Statistical description of the bubble cloud resulting from the injection of air into a turbulent water jet
}

\author{
C. Martínez-Bazán , J.L. Montañés , J.C. Lasheras \\ Departamento de Ingeníeria Mecánica, Area de Mecanica de Fluidos, Universidad Carlos III de Madrid, \\ Avda de la Universidad 30, 28911 Leganés, Madrid, Spain \\ Departamento de Motopropulsión y Termofluidodinámica, ETSI Aeronáuticos, \\ Pza. Cardenal Cisneros 3, 28040 Madrid, Spain \\ Department of Mechanical and Aerospace Engineering, University of California at San Diego, \\ 9500 Gilman Drive, La Jolla, CA 92093-041I, USA
}

\begin{abstract}
The final bubble size distribution, resulting from the break-up of an air jet injected into the central axis of a fully developed, high Reynolds number turbulent water jet has been measured using a Phase Doppler Particle Analyzer (PDPA). The shape of the final size distribution is shown to depend not only on the dissipation rate of turbulent kinetic energy, c, but also on the global void fraction, $x_{\mathrm{H}}$. It has been shown that such a dependence can be expressed as a function of two dimensionless numbers, namely the jet Weber number, $W_{\mathrm{cn}}=\rho U_{\mathrm{J}}^{2} D_{\mathrm{J}} / \sigma$, and the ratio between the initial bubble's size and the critical diameter, $D_{0} / D_{\mathrm{c}}$. The statistical properties of the time and distance separating two bubbles of the same diameter, after the turbulent break process is complete, have also been measured. The probability density function of the interarrival time between two consecutive bubbles was found to follow an exponential distribution with intensity factor, $\lambda$, depending on the number density of bubbles of a certain diameter and on the velocity of the flow.
\end{abstract}

Keywords: Turbulence; Jet; Bubblc; Break-up; Size distribution

\section{Introduction}

The statistical description of the cloud of particles resulting from the break-up of an immiscible fluid immersed into a turbulent flow is of great relevance to many engineering and natural processes such as two-phase flow chemical reactors, gas-liquid separators, liquid atomization and spray 
systems, aeration processes, etc. Of particular interest in all these cases is not only the description of the probability density function (pdf) of the size of the resulting particle cloud, but also of the spatial and temporal variations of the concentration of particles of a given size throughout the cloud.

When a particle is immersed into an immiscible turbulent flow, the turbulent stresses acting on the surface of the particle may surpass the confinement due to the surface tension, causing the particle to break into smaller ones (Kolmogorov, 1949; Baranaev et al., 1949). In an earlier study, Martínez-Bazán et al. (1999a) showed that the characteristic time needed for this break-up depends on the particle size and on the dissipation rate of turbulent kinetic energy of the underlying turbulence, $\epsilon$. We found that for each value of the dissipation rate, there is a particle size, namely $D_{g \max } \approx 2.05(\sigma / \rho)^{3 / 5} \epsilon^{-2 / 5}$, for which the break-up time is a minimum. We also found that this break-up time is not a monotonic function of the particle size. In the limit of very large particles, the confinement of the surface tension becomes negligibly small and the break-up time is given by $t_{\mathrm{b}} \propto D^{2 / 3} \epsilon^{-1 / 3}$, while for particles smaller than $D_{g \max }$, the surface tension stresses dominate, and the break-up time decreases with the size as $t_{\mathrm{b}} \propto(\sigma / \rho)^{2 / 3} \epsilon^{-3 / 5}\left(D / D_{\mathrm{c}}-1\right)^{-1 / 2}$, where $D_{\mathrm{c}} \approx 1.26(\sigma / \rho)^{3 / 5} \epsilon^{-2 / 5}$. In a subsequent study, Martínez-Bazán et al. $(1999 \mathrm{~b})$ also showed that upon break-up, the most probable size of the daughter particles depends on both the initial particle size, $D_{0}$, and the value of the dissipation rate of turbulent kinetic energy.

The existence of a cascade-type break-up process appears to indicate that when a particle of size $D_{0}$ is immersed into a turbulent flow of given characteristics, the final pdf of the resulting particle size distribution should depend on the initial particle size, $D_{0}$, the dissipation rate of turbulent kinetic energy, $\epsilon$, and the residence time (or conversely the number of steps involved in the breakup cascade). Clearly, this cascade-type of break-up implies that there should be two well-differentiated asymptotic regimes. If the residence time, $t_{\mathrm{r}}$, is comparable to the particle break-up time, $t_{\mathrm{b}}$, the particle will undergo a small number of divisions, and the final size pdf should retain a memory of the initial size. However, if the particle break-up time is small compared to the residence time, the cascade will involve many break-up steps resulting in a final pdf whose shape should be determined by the shape of the daughter pdf during the break-up process.

Many natural and engineering systems involve the break-up of a lump of fluid in a turbulent flow with a time varying intensity. An example is the break-up of a lump of fluid immersed into a turbulent jet in a spray atomizer. In this case, as the particles are convected by the mean flow into regions of decaying turbulence, the cascade break-up takes place while they remain in regions of a given $\epsilon$ during a certain residence time. Martínez-Bazán et al. (2000) recently showed that under conditions of decaying turbulence, if the number of break-up steps involved in the cascade is sufficiently large, the final size pdf acquires a universal shape. The important questions of interest which still remain unsolved are: (1) Under what set of conditions (i.e. turbulent intensity, void fraction and residence time) will the final size pdf exhibit a universal shape? (2) what set of parameters determine this shape? and (3) how does the rate of decay of the turbulent kinetic energy of the carrier flow affect the shape of the final pdf?

To answer these questions we have conducted a set of experiments where we have systematically varied the magnitude of the dissipation rate of turbulent kinetic energy at the injection point, the rate of decay of $\epsilon$, and the initial size of the lump of air injected into the flow, $D_{0}$. In addition to measuring the shape of the final bubble size pdf and its dependence on $\epsilon$ and $D_{0}$, we have also measured the statistical properties of the bubble cloud. In particular, we focused our study on the temporal and spatial variations of the bubble concentration throughout the cloud. 


\section{Experimental setup and measurement techniques}

The experiments were performed by continuously injecting an air jet into the fully developed region along the central axis of a high Reynolds number turbulent water jet. The experimental facility is shown in Fig. 1. Since this facility is the same as that used in our previous studies (Martínez-Bazán et al., 1999a,b, 2000), the reader is referred to Martínez-Bazán (1998) for a complete description. We, therefore, will omit unnecessary details here.

Since the volume of water issued by the submerged water jet during the measuring time is very small compared to the volume of the tank and since the dimension of the tank is very large compared to the size of the water nozzle the turbulent water jet in which the break-up was studied can be considered to be a free jet. The air jet was continuously injected into the central axis of the water jet through a hypodermic needle of diameter $D_{\mathrm{n}}=0.394 \mathrm{~mm}$ (see Fig. 1). In order to insure that the properties of the turbulence were fully characterized and nearly homogeneous and isotropic, we limited our studies to cases where the bubbles break-up had been completed at a specified downstream distance from the air injection point and where the bubbles remained in the core of the jet (radial distances less than $1 / 3 L_{\mathrm{J}}$, where $L_{\mathrm{J}}$ is the value of the width of the jet at the

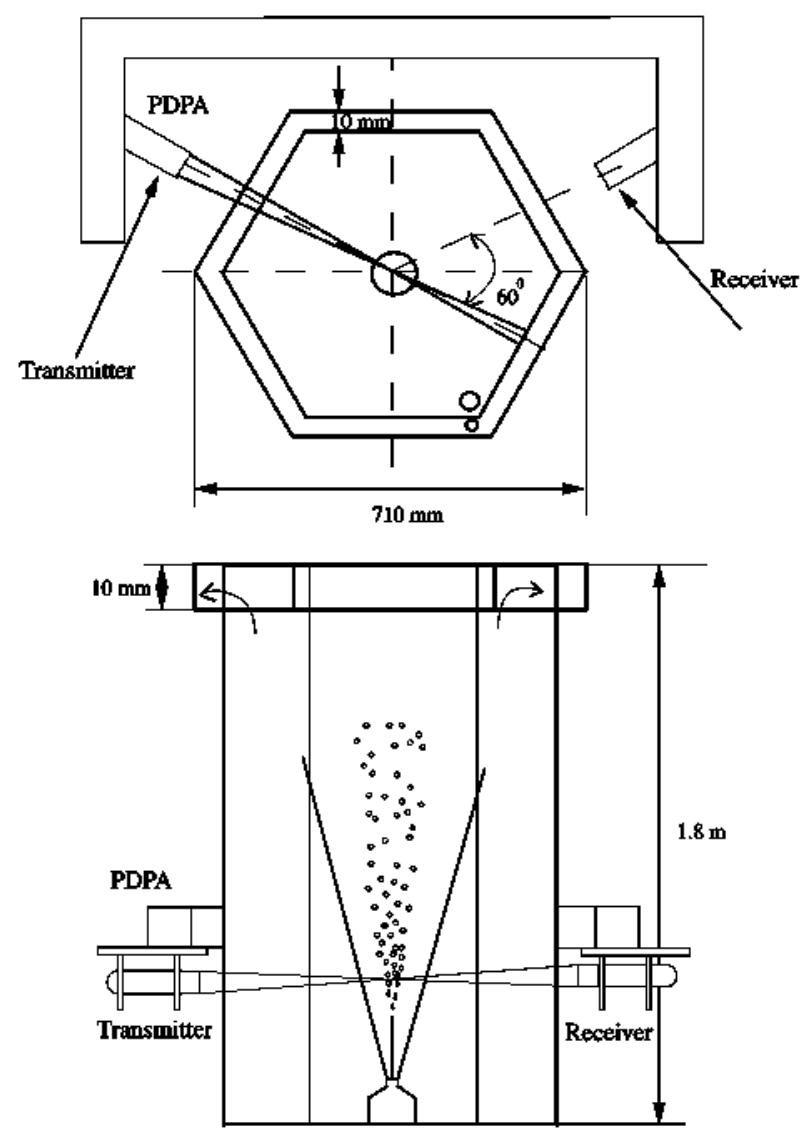

Fig. 1. Experimental facility. 
measuring point). The local values of $\epsilon$ reported in this paper were estimated from hot film measurements performed at the air injection point. An estimate of $\epsilon$ at the central axis of the jet can be given by

$$
\epsilon=15 v \int_{0}^{\infty} k_{1}^{2} E_{11}\left(k_{1}\right) \mathrm{d} k_{1},
$$

where $E_{11}\left(k_{1}\right)$ is the one-dimensional spectrum and $k_{1}$ is the wave number in the direction of the flow. The length of the film used in our experiments, $L_{\mathrm{f}}=1 \mathrm{~mm}$, was larger than the Kolmogorov scale, $L_{\mathrm{f}}>\eta$. Thus, small eddies could not be resolved to perform the integral (1) and an alternative method was applied. The one-dimensional energy spectrum, shown in Fig. 2, was obtained from the temporal series of the velocity measurements. The inertial subrange was identified in the experimentally obtained spectra and, therefore, the value of $\epsilon$ could be extracted through the following power law (Hinze, 1975):

$$
E_{11}\left(k_{1}\right)=\frac{18}{55}\left(\frac{8}{9 \alpha}\right)^{2 / 3} \epsilon^{2 / 3} k_{1}^{-5 / 3}
$$

In Eq. (2) $\alpha=0.452$ is an empirical constant provided by Gibson (1963) and obtained at the axis of a turbulent round jet. Once the inertial range was identified, we fitted the energy spectra given by (2) only to the $-5 / 3$ power law portion of our spectra. Notice that, in Eq. (2), $\epsilon$ is the only adjustable parameter to be determined. The value of $\epsilon$ was, therefore, adjusted until the error obtained during the fitting process was minimized. The solid line in Fig. 2 indicates the best fit of Eq. (2) to the inertial subrange of our spectrum. Measurements of $\epsilon$ along the axis of the jet are depicted in Fig. 3 for two different exit velocities, namely $U_{0}=12 \mathrm{~m} / \mathrm{s}$ and $U_{0}=17 \mathrm{~m} / \mathrm{s}$, which correspond to $R e=36,000$ and $R e=51,000$, respectively. Two sets of experiments at $R e=36,000$ were performed to check the repetitiveness of the measurements.

The kinetic energy of a high Reynolds number turbulent flow cascades down from the largest, integral scale to the smallest, viscous length, until it is eventually dissipated. Therefore, the rate of

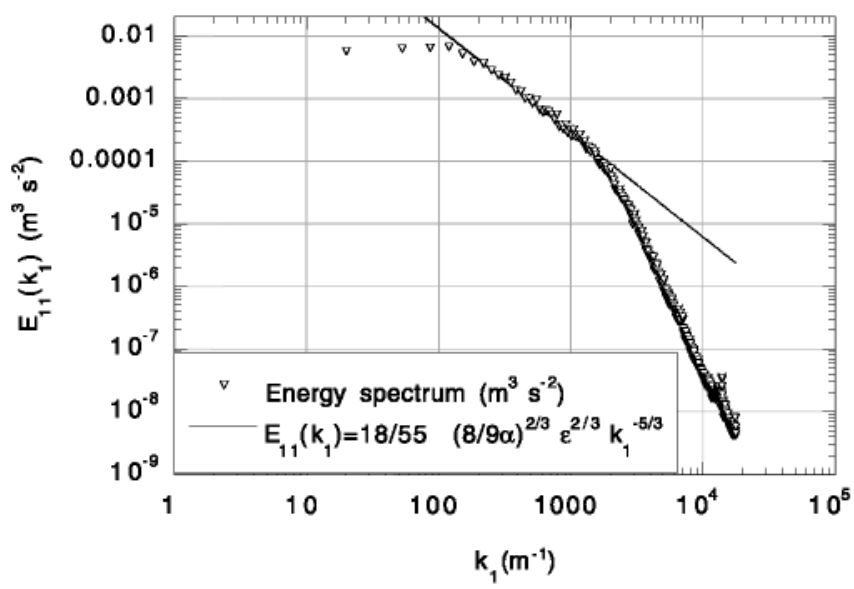

Fig. 2. Experimentally measured energy spectrum. $X / D_{\mathrm{J}}=20, R e=36,000 . D_{\mathrm{J}}$ is the diameter of the water nozzle. 


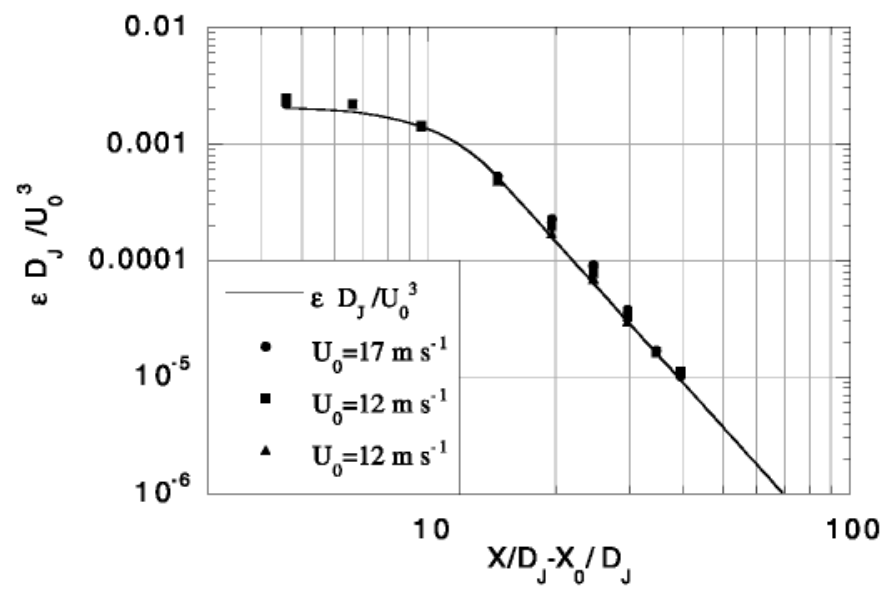

Fig. 3. Normalized dissipation rate of turbulent kinetic energy, $\epsilon D_{\mathrm{J}} / U_{0}^{3}$. Here $X_{0} / D_{\mathrm{J}} \approx 5.4$.

dissipation of turbulent kinetic energy can be estimated through the energy flux across the large scales as (Antonia et al., 1980)

$$
\epsilon \propto \frac{u_{\mathrm{c}}^{3}}{\ell},
$$

where $u_{\mathrm{c}}$ and $\ell$ are the characteristic turbulent velocity and length scale, respectively. Let us define the characteristic velocity as the centerline velocity, $U_{\mathrm{c}}$, and the characteristic length, $\ell=r_{1 / 2}$, as the radial distance at which the velocity is $U_{r_{1 / 2}}=U_{\mathrm{c}} / 2$. Since, in a turbulent round jet, $U_{\mathrm{c}} / U_{0} \propto$ $\left(X / D_{\mathrm{J}}-X_{0} / D_{\mathrm{J}}\right)^{-1}$ and $r_{1 / 2} \propto\left(X-X_{0}\right)$, with $X$ being the downstream distance from the nozzle and $X_{0}$ the virtual origin, Eq. (3) can be expressed as

$$
\frac{\epsilon D_{\mathrm{J}}}{U_{0}^{3}} \propto\left(\frac{X}{D_{\mathrm{J}}}-\frac{X_{0}}{D_{\mathrm{J}}}\right)^{-4} .
$$

This equation was derived by Friehe et al. (1972), and compared to experimental data. The downstream evolution of the normalized rate of dissipation, $\epsilon D_{\mathrm{J}} / U_{0}^{3}$, obtained from our measurements at two different Reynolds numbers, is exhibited in Fig. 3. The solid line represents a correlation of the estimations of $\epsilon$ based on the scaling argument given by Eq. (4). The results presented in Fig. 3 were used in this paper to determine the local values of $\epsilon$.

To measure the bubble size and the axial and radial components of their velocity simultaneously, an Aerometrics Phase Doppler Particle Analyzer (PDPA) was used. The receiver collecting angle was set at $60^{\circ}$ (see Fig. 1) in the forward scatter direction. This gives a linear relationship between the phase difference and the diameter of the scattering bubble, as calculated from analytical modeling using the Lorenz-Mie theory (Bachalo and Houser, 1984; Breña de la Rosa et al., 1989, 1990; Sankar and Bachalo, 1991). In all the cases presented here the measurements were taken at 20 nozzle diameters, 10 diameters downstream from the air injection point. This location was determined to be downstream from the location where the break-up process had already finished. The Reynolds number, $R e=U_{\mathrm{J}} D_{\mathrm{J}} / v$, where $U_{\mathrm{J}}$ is the water velocity at the exit nozzle and $D_{\mathrm{J}}$ is the diameter of the nozzle, was varied from 27,000 to 94,000 giving, 
therefore, values of $\epsilon$ at the air injection point which ranged from $\epsilon \approx 1127 \mathrm{~m}^{2} / \mathrm{s}^{3}$ to $\epsilon \approx 20,780$ $\mathrm{m}^{2} / \mathrm{s}^{3}$. The global air void fraction, defined as $\alpha_{\mathrm{H}}=Q_{\mathrm{a}} /\left(Q_{\mathrm{a}}+Q_{\mathrm{w}}\right)$, where $Q_{\mathrm{a}}$ and $Q_{\mathrm{w}}$ are the flow rates of air and water, respectively, was varied from $2.5 \times 10^{-4}$ to $1.6 \times 10^{-2}$.

\section{Effect of the initial bubble size, $D_{0}$, on the maximum bubble size of the final distribution, $D_{\max }$}

In a previous paper, Martínez-Bazán et al. (2000) found that, in a turbulent water jet, when the diameter of the bubbles was made dimensionless with the Sauter mean diameter, $D_{32}$, of the final bubble size distribution all the $\operatorname{pdf}\left(D / D_{32}\right)$ collapsed onto a single, universal curve. We also showed that the effect of $\alpha_{\mathrm{H}}$ on the shape of the final size pdf cannot be only attributed to an effect of the attenuation of $\epsilon$ due to the presence of the disperse phase. Rather, it must be an additional effect of the size of the initial lump of air, $D_{0}$, injected in the flow. This implies that the only parameter needed to describe the shape of the final bubble pdf is $D_{32 .}{ }^{1}$ Therefore, to completely describe the shape of the final bubble size distribution in a decaying turbulent flow, one only needs to know the dependence of $D_{32}$ on $\epsilon$ and $\alpha_{\mathrm{H}}$ or, equivalently, on $D_{0}$.

The dependence of the maximum bubble size on the local value of the dissipation rate of TKE of the underlying turbulence was found to be consistent with the results of classical models based on the assumption of local isotropy and homogeneous turbulence (Kolmogorov, 1949; Hinze, 1955; Berkman and Calabrese, 1988; Baldyga and Bourne, 1993), which simply predict the existence of a critical maximum size, given by

$$
D_{\max } \propto\left(\frac{\sigma}{\rho}\right)^{3 / 5} \epsilon^{-2 / 5},
$$

where $\sigma$ is the interfacial surface tension, and $\rho$ is the density of the continuous phase. In the case of agitated tanks, where $\epsilon$ remains constant during the break-up process, Hinze (1955) found that ${ }^{2}$

$$
D_{\max }\left(\frac{\sigma}{\rho}\right)^{-3 / 5} \epsilon^{2 / 5}=0.725
$$

although he reported an appreciable dispersion of the experimental data possibly due to inaccurate drop size measurements.

In our previous work, we found that, in a turbulent jet, both the break-up frequency and the size of the daughter bubbles depend on the size of the mother bubble (Martínez-Bazán et al., $1999 \mathrm{a}, \mathrm{b}$ ). Extending Eq. (5) to the case of a decaying turbulent flow, $D_{\max }$ can be expressed as a function of $D_{0}$ and the critical diameter, $D_{\mathrm{c}} \propto(\sigma / \rho)^{3 / 5} \epsilon^{-2 / 5}$ as

$$
D_{\max }=F\left(D_{0}, D_{\mathrm{c}}\right)
$$

\footnotetext{
${ }^{1}$ Or any high moment of the distribution such $D_{90 \%}$.

${ }^{2}$ In his experiments Hinze defined $D_{\max }$ as the value for which $95 \%$ total volume is contained in drops with $D \leqslant D_{\max }$.
} 
and, in dimensionless form,

$$
\frac{D_{\max }}{D_{\mathrm{c}}}=F_{1}\left(\frac{D_{0}}{D_{\mathrm{c}}}\right) .
$$

In a turbulent jet, the value of $\epsilon$ on the central axis decreases with the distance from the nozzle exit. Thus, during the time needed for a bubble of initial size $D_{0}$ to finish its break-up cascade process (which involves several break-up events), it will be transported to regions of decreasing values of $\epsilon$. The rate of decay of $\epsilon$ can be estimated as $\epsilon \propto U_{\mathrm{J}}^{3} / D_{\mathrm{J}}\left(X / D_{\mathrm{J}}-X_{0} / D_{\mathrm{J}}\right)^{-4}$ (Friehe et al., 1972). Eqs. (7) and (8) can, therefore, be expressed as

$$
\frac{D_{\max }}{D_{\mathrm{J}}}=F_{2}\left(D_{0} / D_{\mathrm{c}}\right) W_{\mathrm{en}}^{-3 / 5},
$$

where $W_{\mathrm{en}}=\rho U_{\mathrm{J}}^{2} D_{\mathrm{J}} / \sigma$ is a Weber number based on $D_{\mathrm{J}}$ and $U_{\mathrm{J}}$, and $F_{2}$ is a function which only depends on $D_{0} / D_{\mathrm{c}}$.

To determine the function $F_{2}$ we measured the maximum bubble size, $D_{\max }$, for different cases where the flow rate of air, $Q_{\mathrm{a}}$, and $\epsilon$ at the air injection point were varied to change the initial value of $D_{0}$. The experiments consisted of measurements of the final bubble size distribution, achieved once the break-up process had concluded. In these experiments the needle diameter was kept fixed and equal to $0.394 \mathrm{~mm}$. Three sets of experiments were performed, increasing the root-meansquared velocity of the water jet, $u^{\prime}$, at the air injection point from 1.46 to $5.12 \mathrm{~m} / \mathrm{s}$ for three different values of $Q_{\mathrm{a}}$, namely $Q_{\mathrm{a}}=3.5, Q_{\mathrm{a}}=7.25$, and $Q_{\mathrm{a}}=34.35 \mathrm{ml} / \mathrm{min}$. A final set of experiments was performed by keeping the water velocity constant at $U_{\mathrm{J}}=17.85 \mathrm{~m} / \mathrm{s}\left(u^{\prime}=2.93 \mathrm{~m} / \mathrm{s}\right.$ at the air injection point) while varying the air velocity from 0.35 to $7.73 \mathrm{~m} / \mathrm{s}$. The experimental results obtained in the first three sets of experiments are shown in Fig. 4. Observe that, for very small air injection flow rates $\left(Q_{\mathrm{a}}=3.5 \mathrm{ml} / \mathrm{min}\right.$ and $\left.Q_{\mathrm{a}}=7.25 \mathrm{ml} / \mathrm{min}\right)$, the maximum bubble size decays with a Weber number power law almost equal to the one predicted by the simple theory given in Eq. (9). Fig. 4 also shows that the function $F_{2}$ depends on the initial bubble size,

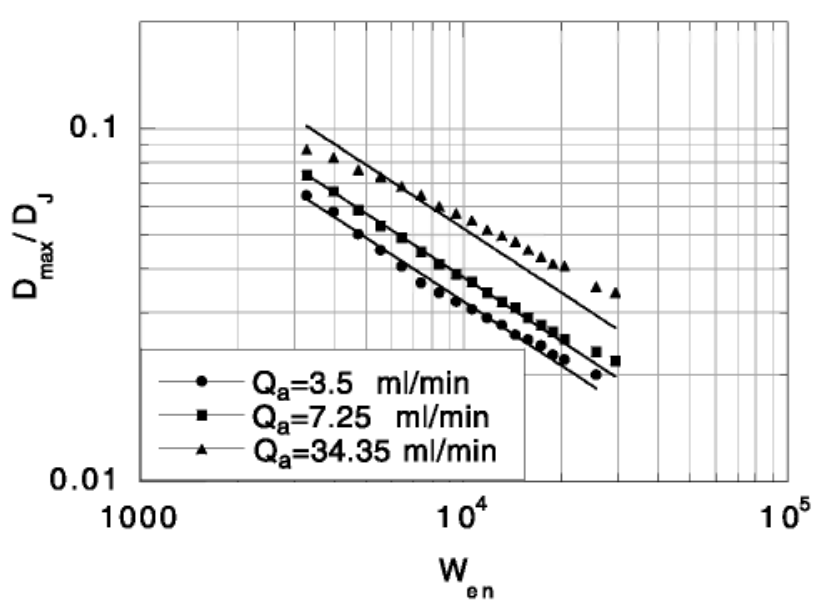

Fig. 4. Variation of the maximum bubble size with the nozzle Weber number. $Q_{a}$ is the flow rate of air injected. Straight lines indicate a $-3 / 5$ decaying power law given in Eq. (9). 
Table 1

Experimental conditions

\begin{tabular}{lllll}
\hline & $U_{\mathrm{w}}(\mathrm{m} / \mathrm{s})$ & $u^{\prime}(\mathrm{m} / \mathrm{s})$ & $U_{\mathrm{a}}(\mathrm{m} / \mathrm{s})$ & $X / D_{\mathbf{j}}$ \\
\hline Set 1 & 4.28 & 1.07 & 4.28 & 20 \\
Set 2 & 2.75 & 0.69 & 2.75 & 29 \\
Set 3 & 2.75 & 0.69 & $>U_{w}$ & 29 \\
\hline
\end{tabular}

$X / D_{\mathbf{J}}$ indicates the air injection point.

$D_{0}$. Notice that the value of $D_{\max }$ increases with the flow rate of air, $Q_{\mathrm{a}}$. Since, as it will be described in Section 3.1, $Q_{\mathrm{a}}$ determines the size of the initial bubble released from the air injection needle, $D_{0}$, it is clear that increasing values of $D_{0}$ result in increasing values of $F_{2}$ and, consequently, larger values of $D_{\max }$. From the measurements of $D_{\max } / D_{\mathrm{J}}$ and $W_{\mathrm{en}}$ obtained for a large range of values of $\epsilon$ and $D_{0}$ one could extract the function $F_{2}\left(D_{0} / D_{\mathrm{c}}\right)$, through the use of Eq. (9), if the size of the initial lump injected into the flow, $D_{0}$, were known.

The value of $D_{0}$ corresponding to the above experimental conditions could not be measured, and had to be extrapolated from the injection conditions. The procedure followed to estimate $D_{0}$ is described below.

\subsection{Dependence of $D_{0}$ on the injection conditions}

In order to determine $D_{0}$ we conducted a series of additional experiments. In these experiments, the turbulent properties of the water jet at the air injection point, $u^{\prime}$, the air flow rate, $Q_{a}$, and the needle diameter, $D_{\mathrm{n}}$, were systematically varied to cover a wide range of flow conditions. To vary the turbulent properties of the water at the air injection point, the Reynolds number of the water jet was fixed at $R e=53,552$ and the air was injected at two different positions, namely $20 D_{\text {J }}$ (Set 1 in Table 1) and $29 D_{1}$ (Set 2) downstream from the water nozzle. In these two sets of experiments the air injection velocity was equal to the local mean velocity of the water jet at each position. In addition, we conducted a third set of experiments with the air injection point fixed at 29 jet diameters (Set 3), but with air injection velocities greater than the local mean velocity of the water jet. See Table 1 for a description of the experimental conditions.

The size of the bubbles releasing from the injection needle was measured by analyzing over 3000 digital images taken with a CCD camera at a very short exposure time of $1 / 80,000 \mathrm{~s}$. A typical image of bubbles detaching from the air injection needle is shown in Fig. 5. The mean bubble diameter was found to increase with both the needle diameter, $D_{n}$, and the air flow rate. Furthermore, the bubble mean diameter was found to decrease with increasing values of the rootmean-square of the water velocity at the air injection point, $u^{\prime}$. The dependence of the Sauter mean diameter, $D_{32}$, of the measured initial bubble size distributions on the needle diameter, $D_{\mathrm{n}}$, is shown in Fig. 6 for different values of $u^{\prime}$ and $Q_{\mathrm{a}}{ }^{3}$

When considering the turbulent break-up of a bubble that can translate freely, it is known that turbulent scales larger than the bubble's diameter simply transport the bubble from one location to another. Scales of the order of the bubble diameter, or smaller, are responsible for the break-

\footnotetext{
${ }^{3}$ Since in our turbulent water jet $u^{\prime} / U_{\mathrm{w}} \approx 25 \%$, the water velocity at the air injection point, $U_{\mathrm{w}}$, was chosen to describe the experimental conditions.
} 


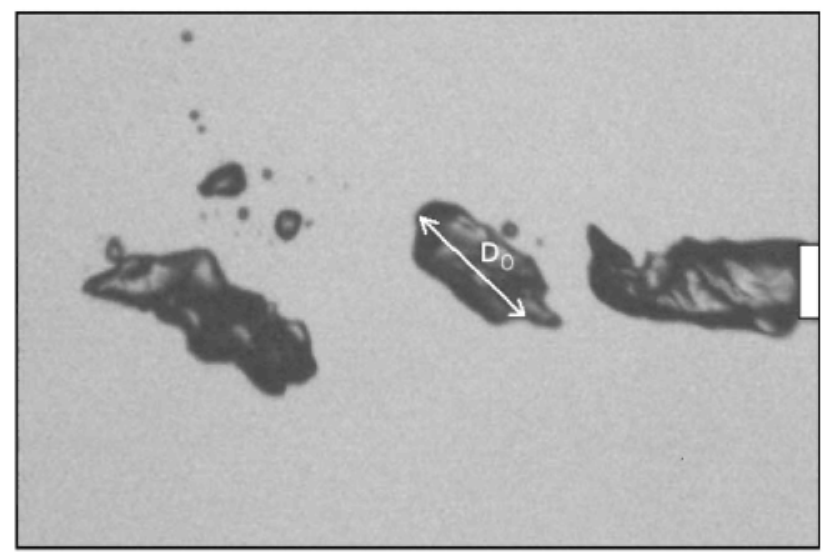

Fig. 5. Characteristic initial bubble size, $D_{0}$. Injection point $X / D_{\mathrm{J}}=29, U_{\mathrm{a}}=U_{\mathrm{w}}=2.75 \mathrm{~m} / \mathrm{s}, D_{\mathrm{n}}=0.838 \mathrm{~mm}$. Flow goes from right to left. The air injection needle exit is shown in white.

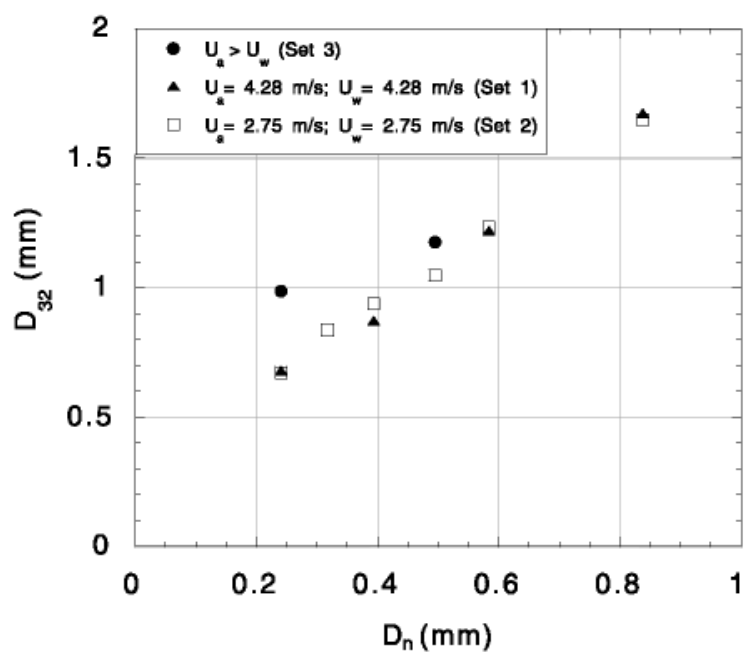

Fig. 6. Measured Sauter mean diameter versus needle diameter. $R e=53,552$.

up. For bubbles in the inertial subrange, this means that their characteristic break-up time is given by $t_{\mathrm{b}} \propto\left(D^{2} / \epsilon\right)^{1 / 3}$. In the present scenario, the initial lump of air injected into the turbulent water flow cannot freely translate. Indeed, it is attached to the injection needle. In this case, large scales can sweep the initial lump of air off of the injection needle. Additionally, smaller scales may also break the initial lump, as in the case of a free bubble. Therefore, in the initial break-up problem, all turbulent scales must be considered. This implies that the characteristic turbulent break-up time should be given by $t_{\mathrm{b}} \propto D_{\mathrm{n}} / u^{\prime}$ rather than $t_{\mathrm{b}} \propto\left(D_{\mathrm{n}}^{2} / \epsilon\right)^{1 / 3}$. The diameter of the bubbles resulting from the initial break-up can, consequently, be estimated as $D_{0} \propto\left(Q_{\mathrm{a}} t_{\mathrm{b}}\right)^{1 / 3}$ giving

$$
D_{0} \propto\left(\frac{6}{\pi} \frac{Q_{\mathrm{a}} D_{\mathrm{n}}}{u^{\prime}}\right)^{1 / 3}=\left(\frac{3}{2} \frac{U_{\mathrm{a}}}{u^{\prime}}\right)^{1 / 3} D_{\mathrm{n}}
$$




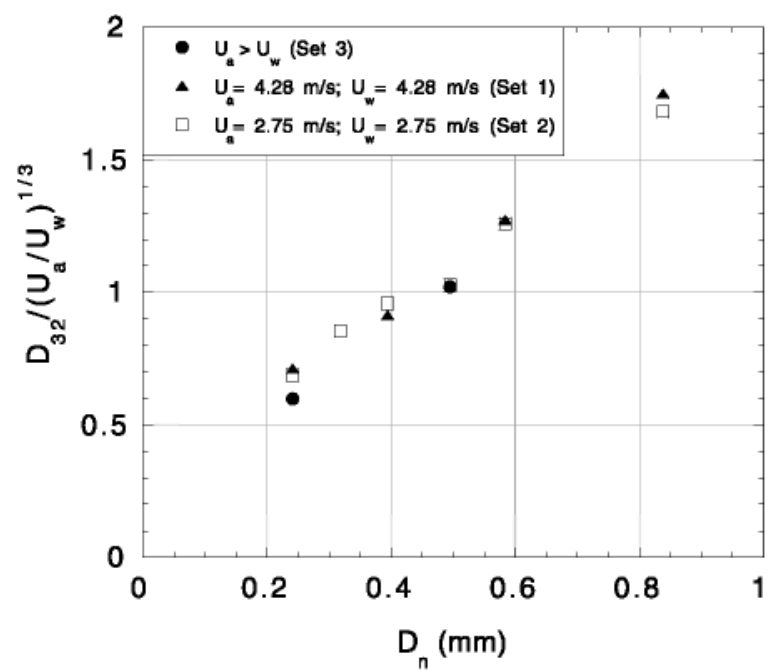

Fig. 7. Dependence of $D_{32} /\left(U_{\mathrm{a}} / U_{\mathrm{w}}\right)^{1 / 3}$ with the needle diameter. $R e=53,552$.

In our experiments, when the air velocity was equal to the local value of the water jet mean velocity, $U_{\mathrm{w}}$, the above equation simplified to, ${ }^{4} D_{0} \propto D_{\mathrm{n}}$, a linear dependence shown in Fig. 6 . Notice that for the cases where $U_{\mathrm{a}}=U_{\mathrm{w}}$, the Sauter mean diameter depends only on $D_{\mathrm{n}}$, even though we varied the value of the dissipation rate of TKE from $\epsilon=208 \mathrm{~m}^{2} \mathrm{~s}^{-3}$ to $\epsilon=$ $1205 \mathrm{~m}^{2} \mathrm{~s}^{-3}$. Also note that the values of $D_{32}$ measured for the two cases where $U_{\mathrm{a}}>U_{\mathrm{w}}$ (filled circles, •, in Fig. 6) are larger than those obtained with $U_{\mathrm{a}}=U_{\mathrm{w}}$ for the same needle diameter. This behavior confirms the estimated diameter given by Eq. (10). Eq. (10) can also be expressed as

$$
\frac{D_{0}}{\left(U_{\mathrm{a}} / u^{\prime}\right)^{1 / 3}}=\beta D_{\mathrm{n}},
$$

where $\beta$ is a constant or order one to be determined experimentally. This simple model is verified in Fig. 7, where we have plotted $D_{32} /\left(U_{\mathrm{a}} / u^{\prime}\right)^{1 / 3}$ versus $D_{\mathrm{n}}$. From this figure a value of the constant $\beta \approx 1.4$ can be inferred.

We now have a simple model to determine $D_{0}$ as a function of the injection conditions, $u^{\prime}, U_{\text {a }}$ and $D_{\mathrm{n}}$. Using Eq. (11) to estimate the value of $D_{0}$ for the experimental conditions at which the final bubble pdfs were measured, and combining with Eq. (9) to calculate $F_{2}$, we have been able to obtain the dependence of $F_{2}$ on $D_{0} / D_{\mathrm{c}}$, and consequently, the dependence of $D_{\max } / D_{\mathrm{J}}$ on $D_{0} / D_{\mathrm{c}}$. Fig. 8 portrays the evolution of $F_{2}$ with $D_{0} / D_{\mathrm{c}}$. In this figure Eq. (11) was used to estimate the values of $D_{0}$ for each experimental case, and $D_{\mathrm{c}}$ was calculated using the experimentally measured, local values of $\epsilon$ at the air injection point. Fig. 8 also shows that the dependence of $F_{2}$ on $D_{0} / D_{\mathrm{c}}$ is almost linear, giving an almost perfect collapse of all the experimental data onto the same line.

Summarizing the above results, we have shown that the final bubble size pdf depends on the value of $\epsilon$ at the air injection point, the size of the initial bubble, $D_{0}$, and the residence time of the

\footnotetext{
${ }^{4}$ In Figs. 6 and 7, the Sauter mean diameter, $D_{32}$, of the initial distribution of bubbles was selected as a measure of $D_{0}$.
} 


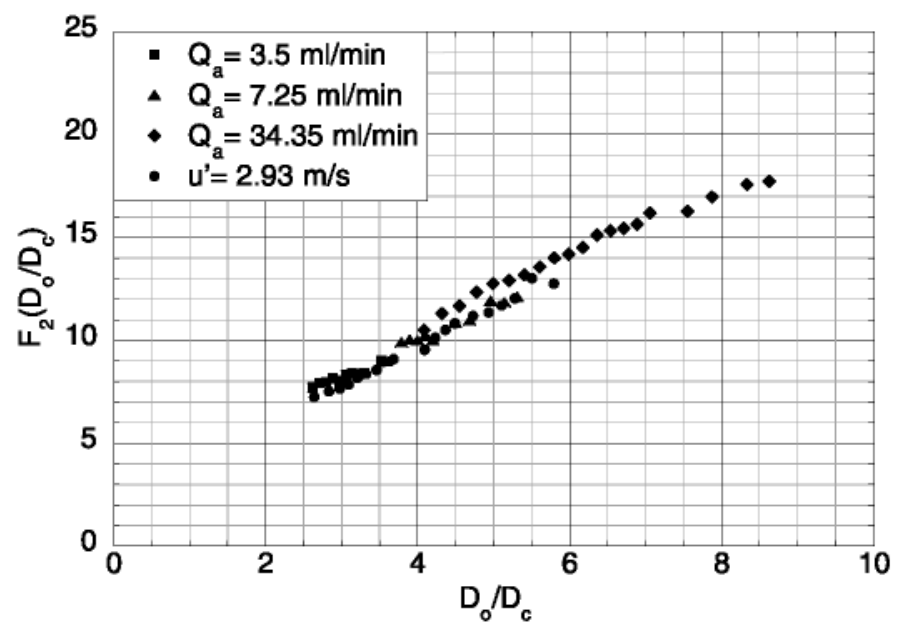

Fig. 8. Evolution of $F_{2}$ with $D_{0} / D_{\mathrm{c}}$.

bubble in regions of active $\epsilon$. Martínez-Bazán et al. showed that, for sufficiently large values of $\epsilon$, when the diameter is made dimensionless with $D_{32}$, all the final pdfs collapsed onto the same curve. Therefore, the final value of $D_{32}$ is the only parameter needed to characterize the shape of the final bubble pdf. Finally $D_{32}$ is given by Eq. (9) where $F_{2}$ is linearly dependent on $D_{0} / D_{\text {c }}$.

Up to this point we have focused our analysis to study the shape of the final bubble size distribution. In the following section we will describe the concentration of bubbles throughout the spray and its dependence on $\epsilon$ and the bubble size.

\section{Inter-arrival time between bubbles}

Since we are able to measure not only the size of the bubbles crossing the probe volume but also their velocity and their arrival time, we can compute the time and, consequently, the distance separating two consecutive bubbles of the same size. This enables us to analyze the statistics of the fluctuations of the concentration of bubbles and their dependence on the bubble size, $D, \epsilon$ and $\alpha_{\mathrm{H}}$. Fig. 9 shows a typical time series of the bubble size diameter measurements of our experiments. Simple inspection of this data indicates that there could be some clustering of the occurrence times of the bubbles. This type of information can be very useful in order to study clustering events of bubbles of different sizes or to infer if the formation frequencies of bubbles respond to certain frequencies of the flow. One would expect that bubbles of similar diameter behave similarly in terms of formation and dispersion throughout the cloud. In the range of bubble sizes of interest here, the bubble Stokes number, defined as

$$
S t=\frac{D^{2} u^{\prime}}{36 v \ell}
$$

where $D$ is the diameter of the bubble, $u^{\prime}$ and $\ell$ are the characteristic velocity and length scales, and $v$ is the kinematic viscosity, is very small $(S t \ll 1)$. All bubbles will disperse in a similar manner and the effect of any clustering in the time series will only be the consequence of the break-up 


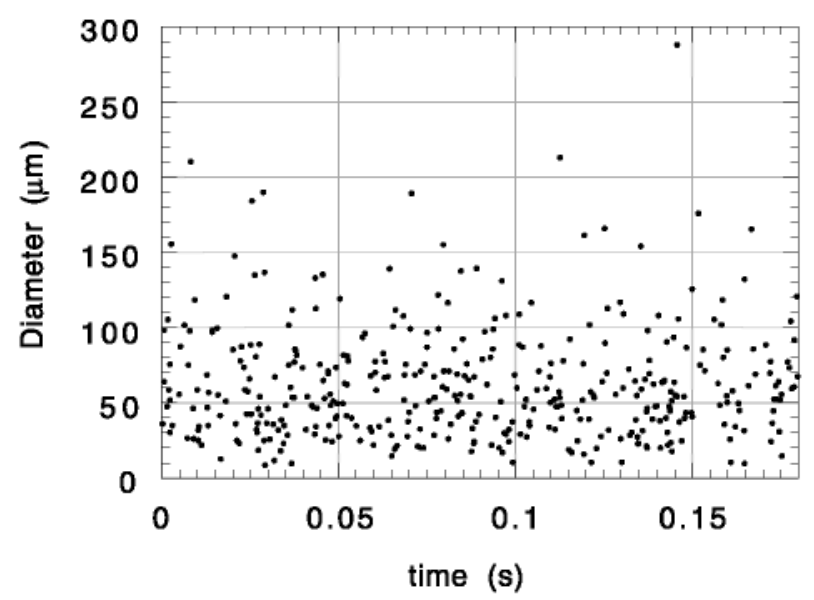

Fig. 9. Time record of bubbles generated by a water jet. $R e=53,552$.

process. In general, the integral length scale of the considered turbulent flow, defined as (Hinze, 1975),

$$
\ell=\frac{\pi E_{11}\left(k_{1}=0\right)}{2 \overline{u^{2}}}
$$

is taken as the characteristic length scale in Eq. (12). $E_{11}\left(k_{1}=0\right)$ is the one-dimensional energy spectrum evaluated at the wave number $k_{1}=0$ and $u^{\prime}$ is the fluctuating axial velocity.

The group of clusters, shown in Fig. 9 by the concentration of a larger number of bubbles within some intervals of time, may indicate the presence of a bubble concentration-time correlation or it may simply be the consequence of a random occurrence. In the analysis of the bubble size pdf (described in the previous sections), the time dependence of the process was not addressed. In the following we will present some experimental measurements of the time series and we will attempt to establish if the break-up of bubbles by a turbulent flow contains some sort of time correlated behavior.

In order to investigate the existence of a time correlation in the bubble break-up process, we subdivided the bubble sizes into classes. For this purpose, the size distribution was discretized into five size-bins, namely $3 \mu \mathrm{m}<D<20 \mu \mathrm{m}, 40 \mu \mathrm{m}<D<60 \mu \mathrm{m}, 80 \mu \mathrm{m}<D<100 \mu \mathrm{m}$, $120 \mu \mathrm{m}<D<200 \mu \mathrm{m}$, and $D>200 \mu \mathrm{m}$. The time separating the arrival of two consecutive bubbles which belong to the same class was recorded. This analysis provided us with information on the temporal variation of concentration of bubbles of different sizes. The probability density functions of the inter-arrival time corresponding to the four size bins are given in Fig. 10 for the case of $R e=53,552$. The distribution corresponding to the fifth size-bin, $D>200 \mu \mathrm{m}$, is not represented due to the very small number of bubbles contained in this size-bin. Notice that for the four size-bins presented, the probability of the inter-arrival time between two consecutive bubbles increases as the inter-arrival time decreases. Fig. 10 shows that there is not a predominant time separating two consecutive bubbles of the same size. Therefore the inter-arrival time pdf does not show a high concentration of bubbles at a characteristic frequency. Imagine, for example, a flow in which bubbles of size $D_{1}$ are formed at a fixed frequency of $100 \mathrm{~Hz}$. If we calculated the pdf of 


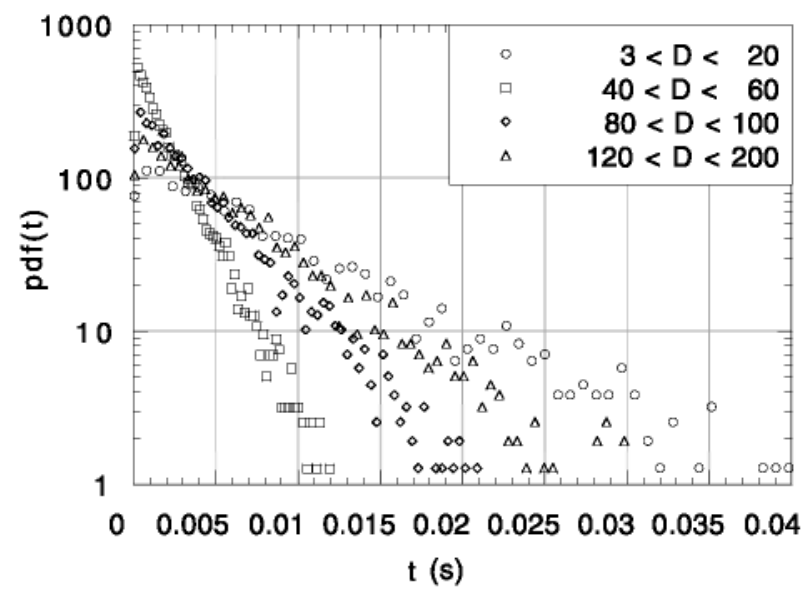

Fig. 10. Inter-arrival time pdf of different classes of bubbles. Re $=53,552$. Symbols are: (O) $3 \mu \mathrm{m}<D<20 \mu \mathrm{m}$, () $40 \mu \mathrm{m}<D<60 \mu \mathrm{m},(\diamond) 80 \mu \mathrm{m}<D<100 \mu \mathrm{m},(\Delta) 120 \mu \mathrm{m}<D<200 \mu \mathrm{m}$.

the time between two consecutive bubbles of size $D_{1}$ we would find a strong peak in the distribution for a value of $t$ equal to $10 \mathrm{~ms}$. Although the data presented in Fig. 10 correspond to an intermediate $R e=53,552$, it can be clearly seen that the pdf follows exponential distributions of different mean value, $t_{\mathrm{m}}=1 / \lambda$,

$$
\operatorname{pdf}(t)=\lambda \mathrm{e}^{-\lambda t},
$$

where $\lambda$ is the intensity factor and $t_{\mathrm{m}}=1 / \lambda$ is the mean as well as the root-mean-square value of the distribution. The slopes of the tails represent the mean rate of the arrival of bubbles of each size class. Note that if the inter-arrival time is scaled with the mean arrival time $t^{*}=t / t_{\mathrm{m}}=\lambda t$,

$$
\operatorname{pdf}\left(t^{*}\right)=\mathrm{e}^{-t^{\star}},
$$

all the pdfs should collapse onto a single one.

The probability density functions shown in Fig. 10, normalized by the mean value, $t^{*}=$ $t / t_{\mathrm{m}}=\lambda t$, are shown in Fig. 11. As indicated by Eq. (15), when using the dimensionless time, $t^{*}$, the $\operatorname{pdf}\left(t^{*}\right)$ follows the same exponential distribution with slope -1 . As one would expect, since the exponential distribution provides the probability for the time between successive events occurring in a Poisson process, it can be concluded that the process of formation of bubbles by a high intensity homogeneous turbulence is a random process which can be well represented by a Poisson distribution. This indicates that individual bubbles within the turbulent jet act independently of each other and, therefore, the movement of one of them is not conditioned by the neighboring ones. In a denser case, where the motion of a bubble may influence the others, this general conclusion is not expected to be valid and the exponential approximation may not apply.

\subsection{Effect of the turbulent kinetic energy on the inter-arrival time}

The time between two consecutive particles which belong to the same size-bin can be defined as

$$
\Delta t_{i}=\frac{1}{C_{i} U_{i} A},
$$




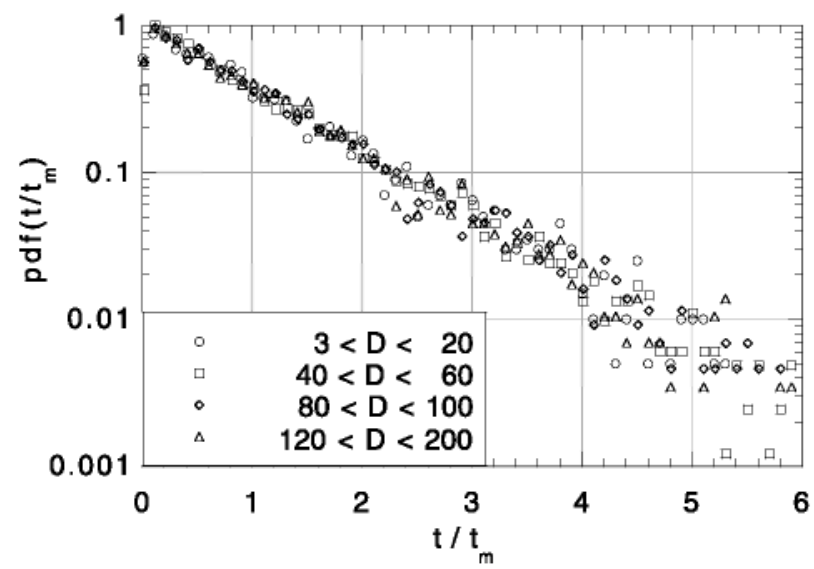

Fig. 11. Pdf of the normalized inter-arrival time, $t / t_{\mathrm{m}}\left(t_{\mathrm{m}}=\right.$ mean inter-arrival time $)$ of different classes of bubbles.

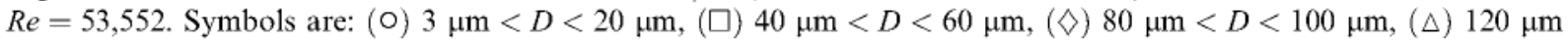
$<D<200 \mu \mathrm{m}$.

where $C_{i}$ and $U_{i}$ are the number of bubbles of size-class $i$ per unit volume and the velocity of a bubble sized in class $i$, respectively, and $A$ is the jet cross-section. Therefore, one would expect that the inter-arrival time, $\Delta t_{i}$, should increase with decreasing values of the concentration, $C_{i}$, and of the velocity, $U_{i}$.

To study the dependence of the inter-arrival time on the Reynolds number of the water jet, or in our case on $\epsilon$, we varied $R e$ by changing the velocity at the exit of the nozzle. The evolution of the mean inter-arrival time of the five bubble size-bins defined above is shown in Fig. 12. As the mean water jet velocity, $U$, increases, the Reynolds number of the jet increases and so does $\epsilon$. Therefore, in addition to increasing the velocity of the bubbles, $U_{i}$, the simultaneous increase in the turbulent kinetic energy of the water flow may also increase the total number of bubbles formed due to increased break-up. This holds true for small sized bubbles which belong to class $1(0)$, class $2(\square)$, and class $3(\diamond)$. Large bubbles within class $5(\boldsymbol{\nabla})$, increase their inter-arrival time since, although their velocity increases, this type of bubbles are more likely to break for larger values of $\epsilon$. Bubbles of size class $4(\Delta)$, lessen their inter-arrival time when the water jet Reynolds number is increased up to a certain value. When this value is reached, the concentration of this bubble class, $C_{4}$, decreases due to increased break-up and the time between two consecutive bubbles begins to increase. The behavior of the standard deviation of the inter-arrival time, although not shown here, is identical to that of the mean value. In fact, it has been found that the ratio of the rms to the mean value, $t^{\prime} / t_{\mathrm{m}}$, is one in all cases, as expected from exponential distributions.

To study the effect of the water jet Reynolds number (or $\epsilon$ ) on the shape of the inter-arrival time pdf, we have selected the bubble size class $2,40 \mu \mathrm{m}<D<60 \mu \mathrm{m}$. Their inter-arrival time pdf is plotted in Fig. 13 for three different values of the water jet Reynolds number. The results are consistent with those shown in Fig. 12. As $R e$ is increased, the inter-arrival time of the class 2 bubbles decreases since their convective velocity is faster and more bubbles are produced due to the break-up of larger ones. Selecting a non-dimensional variable of time, $t / t_{\mathrm{m}}\left(t_{\mathrm{m}}\right.$ is the interarrival mean value), the probability density function, $\operatorname{pdf}\left(t / t_{\mathrm{m}}\right)$, has a self-similar behavior that can be perfectly represented by an exponential distribution, $\operatorname{pdf}\left(t / t_{\mathrm{m}}\right)=\exp \left[-t / t_{\mathrm{m}}\right]$, as shown in 


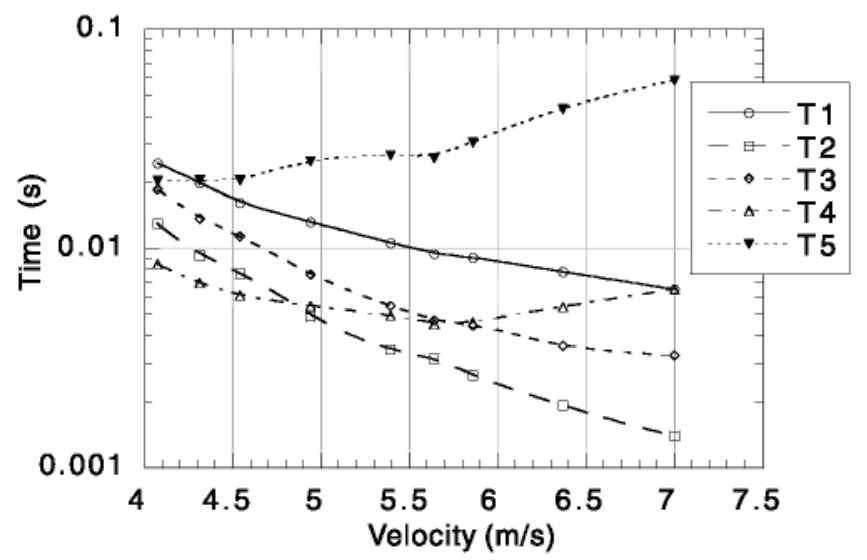

Fig. 12. Evolution of mean inter-arrival time, $t_{\mathrm{m}}$, with the local convective velocity, $U$. The Reynolds number of the jet has been varied from $R e \approx 32,000$ to $R e \approx 60,000$. Symbols are: (O) $3 \mu \mathrm{m}<D<20 \mu \mathrm{m}$, ( $\square$ ) $40 \mu \mathrm{m}<D<60 \mu \mathrm{m}$, $(\diamond) 80 \mu \mathrm{m}<D<100 \mu \mathrm{m},(\Delta) 120 \mu \mathrm{m}<D<200 \mu \mathrm{m},(\mathbf{\nabla}) D>200 \mu \mathrm{m}$.

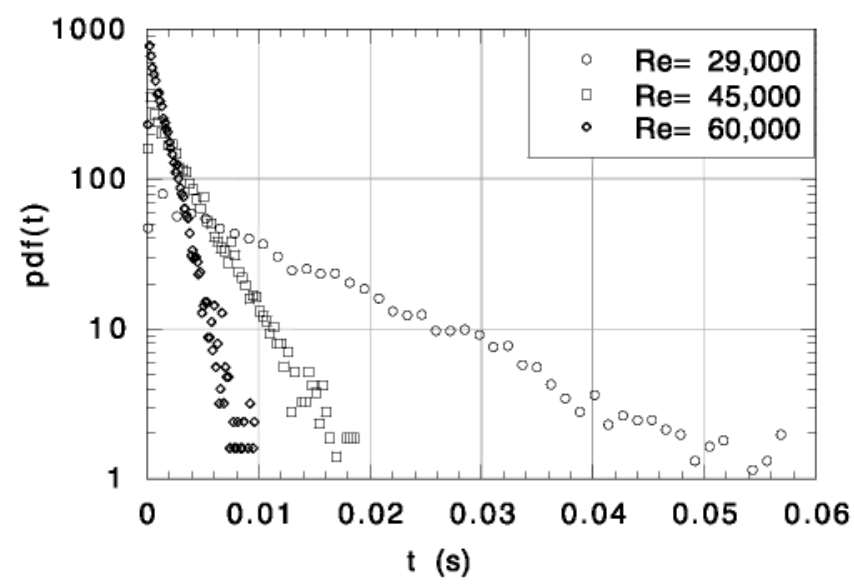

Fig. 13. Evolution of the inter-arrival time probability density function of bubbles of size $40 \mu \mathrm{m}<D<60 \mu \mathrm{m}$ with the Reynolds Number.

Fig. 14. The self-similar behavior is independent of the Reynolds number and of the diameter of the bubble, and the possible clustering mentioned in the beginning of this section is, therefore, the product of a random process.

\section{Inter-arrival distance between bubbles}

The distance between two consecutive bubbles of the same class is calculated from their corresponding inter-arrival time, $\Delta t_{i}$, and their convective velocity, $U_{i}$, as 


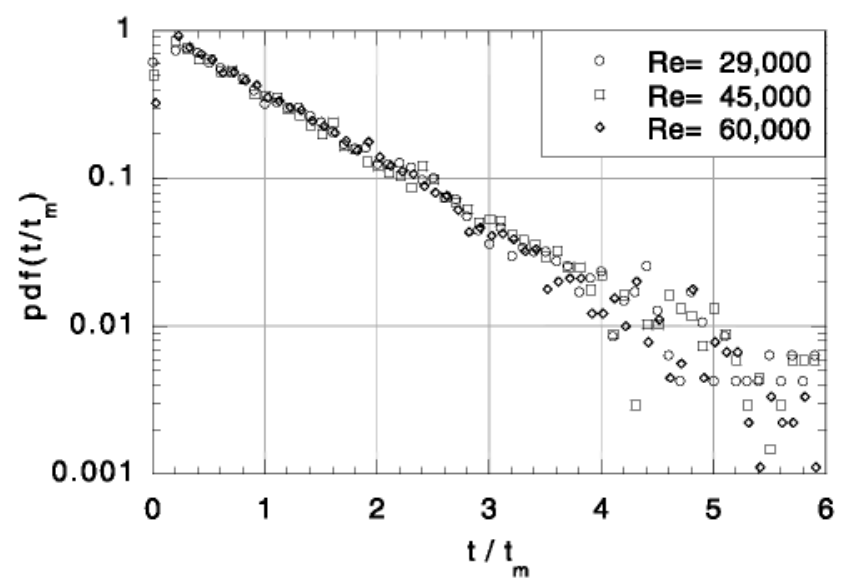

Fig. 14. Evolution of the normalized inter-arrival time pdf for bubbles of size $40 \mu \mathrm{m}<D<60 \mu \mathrm{m}$ with the Reynolds number.

$$
\Delta l_{i}=\Delta\left(t_{i} U_{i}\right)=\frac{1}{C_{i} A} .
$$

The probability density function of the distance separating two consecutive bubbles of the same size follows the same characteristics as those mentioned for the inter-arrival time. Since bubbles of the same class move at the same velocity, the pdf of the inter-arrival distance of bubbles within the same class follows an exponential distribution with an intensity parameter, $\lambda_{l}$, which only depends on the number (concentration) of particles of a certain class $i, C_{i}$. Fig. 10, in the previous section, shows that the steepest slope of the inter-arrival time pdf corresponds to the bubble-size class 2 $(40 \mu \mathrm{m}<D<60 \mu \mathrm{m})$, followed by bubble-size class $3(80 \mu \mathrm{m}<D<100 \mu \mathrm{m})$, and bubble sizeclass $4(120 \mu \mathrm{m}<D<200 \mu \mathrm{m})$. Finally, the least steep slope corresponds to bubble-size class 1 $(3 \mu \mathrm{m}<D<20 \mu \mathrm{m}$ ). The same trend is applicable to the pdf of the inter-arrival distance (not shown here), $\Delta l_{i}=\Delta\left(t_{i} U_{i}\right)$, indicating that the convective velocities of all bubble-size classes, $U_{i}$, are the same and equal to those of the mean flow.

From the above results on the inter-arrival time and inter-arrival distance, we observe that the bubbles are not formed in a predictable manner. A bubble of initial size $D_{0}$ undergoes many break-up events, leading to a wide range of bubble sizes in their final size distribution. It is evident from our measurements that these bubbles are both created and dispersed randomly. Edwards and Marx (1995) developed a theoretical framework for the analysis of the time-based statistics of sprays. Based on their approach, the measured bubbly jet behaves as an ideal spray driven by many superposed Poisson processes (of bubble sizes), each one characterized by a continuous intensity function. All bubbles produced by the break-up of an air mass injected into a turbulent water jet are created and transported downstream in the same way. The pdf of the inter-arrival time or distance between two consecutive bubbles of the same class follows the exponential distribution, characteristic of steady-state case developed by Edwards and Marx (1995). Therefore, all bubbles, in the range of bubble sizes and jet Reynolds numbers presented here, feel the influence of the water jet, which, at its axis, can be characterized as isotropic and homogeneous turbulent flow. Measurements at the edge of the water jet would indicate a preferential concen- 
tration of those bubbles transported by large scales. These results would be shown in the above pdf as a strong deviation from their exponential distribution. Similar types of measurements have been presented by Edwards and Marx (1995), for very small particles, which follow the flow, measured in the internal shear layer of a kerosene spray flame.

To compare our experimental data with a random process we have generated two different sequences of numbers using a random generator. The first sequence was generated by producing a set of equally distributed numbers. The second set was produced using a set of normally distributed numbers. After sorting the sequences in ascending order we calculated the difference between two consecutive numbers and the probability density function of the computed differences. The pdfs obtained with both series, shown in Fig. 15, are identical to those obtained when

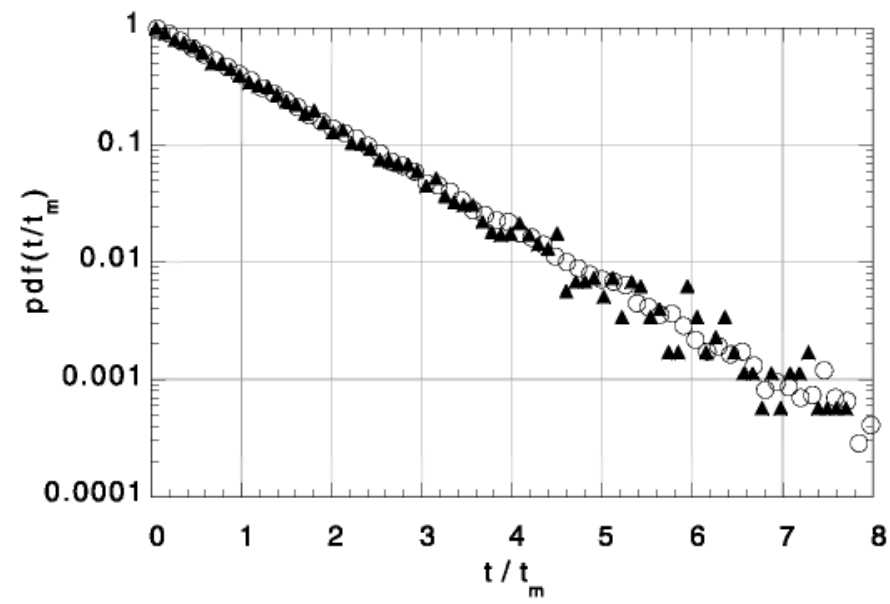

Fig. 15. Pdf of two random signals. Symbols are: (o) uniformly distributed random function, and ( $\mathbf{\Lambda})$ normally distributed random function.

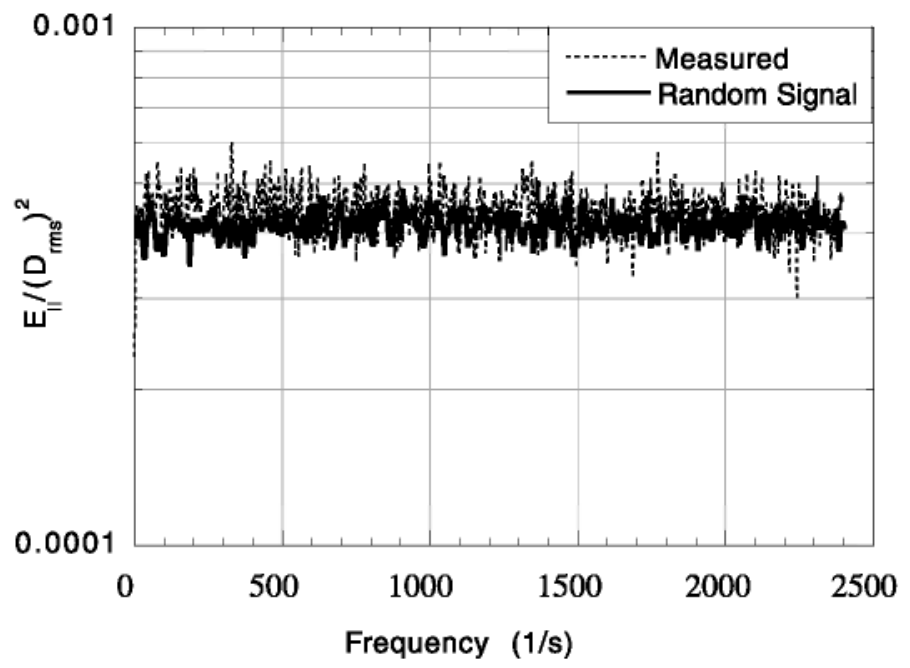

Fig. 16. Power spectrum of a random signal. 
we calculated the inter-arrival time and inter-arrival distance between two bubbles of the same size family. Similarly, we created a random sequence of numbers between 3 and 400 (which corresponds to the size of the measured bubbles) and calculated the power spectrum. The spectrum obtained is presented in Fig. 16 together with the spectrum measured experimentally. Notice that both spectra are uniform with no peaks. Thus, there is no periodic interaction between turbulence and bubble production.

\section{Conclusion}

The shape of the final bubble size pdf, achieved once the break-up process taking place along the central axis of a turbulent water jet is complete, has been studied experimentally. It has been shown that in a turbulent decaying flow, the shape of the bubble pdf depends on the global air void fraction, $\alpha_{\mathrm{H}}$, on the local value of $\epsilon$, and on the ratio between the residence time and the break-up time. The maximum bubble size, $D_{\max }$, has also been measured. The measurements have been found to be consistent with previous models based on ideas of local homogeneous and isotropic turbulent flow. In addition, in a turbulent water jet $D_{\max }$ has been found to have a linear dependence on the initial bubble size, $D_{0}$.

To study the fluctuations of the concentration of bubbles and its dependence on the bubble size and $\epsilon$, the probability density function of the distance between two consecutive bubbles of the same size has also been measured. The inter-arrival time and inter-arrival distance pdf have been shown to follow exponential distributions. This indicates that the process of the break-up and dispersion of bubbles by a high Reynolds number turbulent water jet is a random process given by a Poisson distribution.

\section{Acknowledgements}

The authors gratefully acknowledge the support of the ONR under contract \# N00014-961-0213 and \# N00014-7-1-0305, and the Spanish Ministry of Education (PB98-0142-C04-02). The assistance of Craig Eastwood in some of the experiments is also greatly acknowledged.

\section{References}

Antonia, R.A., Satyaprakash, B.R., Hussain, A.K.M.F., 1980. Measurements of dissipation rate and some other characteristics of turbulent plane and circular jets. Physics of Fluids 23, 695-700.

Bachalo, W.D., Houser, M.J., 1984. Phase/doppler spray analyzer for simultaneous measurements of drop size and velocity distributions. Optical Engineering 23, 583-590.

Baldyga, J., Bourne, J.R., 1993. Drop breakup and intermittent turbulence. Journal of Chemical Engineering of Japan 26, $738-741$.

Baranaev, M.K., Tevenovskiy, Ye.N., Tregubova, E.L., 1949. On the measure of minimal fluctuations in a turbulent flow. Doklady Akademii Nauk SSSR 66, 821-824.

Berkman, P.D., Calabrese, R.V., 1988. Dispersion of viscous liquids by turbulent flow in a static mixer. AIChE Journal $34,602-609$. 
Breña de la Rosa, A., Sankar, S.V., Carr, D.L., Smitl, J.N., Bachalo, W.D., 1990. A study on the morphology of spheroidal bubbles using light scattering interferometry. In: 2nd Congres Francophone de Velocimetrie Laser, Meudon, France.

Breña de la Rosa, A., Sankar, S.V., Weber, B.J., Wang, G., Bachalo, W.D., 1989. A theoretical and experimental study of the light characterization of bubbles using light scattering interferometry. In: International Symposium on Cavitation Inception, ASME Winter Annual Meeting, vol. 89, pp. 63-72.

Edwards, C.F., Marx, K.D., 1995. Multipoint statistical structure of the ideal spray, part I: Fundamental concepts and the realization density. Atomization and Sprays 5, 435-455.

Friehe, C.A., Van Atta, C.W., Gibson, C.H., 1972. Jet turbulence: dissipation rate measurements and correlations. In: Turbulent Shear Flows, AGARD Conference Proceedings, vol. 93, pp. 18-21.

Gibson, M.M., 1963. Spectra of turbulence in a round jet. Journal of Fluid Mechanics 15, 161-173.

Hinze, J.O., 1955. Fundamentals of the hydrodynamics mechanisms of splitting in dispersion process. Journal of AIChE 1, 289-295.

Hinze, J.O., 1975. Turbulence, second ed. MoGraw-Hill, New York.

Kolmogorov, A.N., 1949. On the breakage of drops in a turbulent flow. Doklady Akademii Nauk SSSR 66, 825-828.

Martínez-Bazán, C., 1998. Splitting and dispersion of bubbles by turbulence. Ph.D. Thesis, University of California, San Diego.

Martínez-Bazán, C., Montañes, J.L., Lasheras, J.C., 1999a. On the breakup of an air bubble injected into a fully developed turbulent flow, part I: Breakup frequency. Journal of Fluid Mechanics 401, 157-182.

Martínez-Bazán, C., Montañes, J.L., Lasheras, J.C., 1999b. On the breakup of an air bubble injected into a fully developed turbulent flow. part II: Size pdf of the resulting daughter bubbles. Journal of Fluid Mechanics 401, $183-207$.

Martínez-Bazán, C., Montañes, J.L., Lasheras, J.C., 2000. Bubble size distribution resulting from the breakup of an air cavity injected into a turbulent water jet. Physics of Fluids 12, 145-148.

Sankar, S.V., Bachalo, W.D., 1991. Response characteristics of the phase doppler particle analyzer for sizing spherical particles larger than the light wavelength. Applied Optics 30, 1487-1496. 